\title{
Fundamental analysis of liquid breakup mechanism in a rotary atomizer with square discharge orifice
}

\author{
M. Ghorbanhosseini, S. Rezayat, M. Farshchi* \\ Department of Aerospace Engineering, Sharif University of Technology, Tehran, Iran \\ ${ }^{*}$ Corresponding author: Farshchi@shrarif.edu
}

\begin{abstract}
An experimental investigation of breakup mechanism in a rotary atomizer with square shape discharge orifice at ambient condition has been performed. The effects of a high aspect ratio noncircular discharge channels, particularly a square shape discharge channel, are considered. The motivation of this study is the use of this type of orifice in some small gas turbine engines as well as non-existing observation in literature concerning about high aspect ratio of discharge channel. Visualization experiments are conducted by high speed shadowgraph imaging technique with pulsed light illumination for the first time. The effects of rotational speed and volume flow rate are studied on the breakup structure. The visualizations indicates that the liquid film formed along the channel is pushed to one side of it due to Coriolis force which is dominant in this type of atomizer. Accordingly a crescent shaped liquid film is formed at the square channel exit covering two corners of the square, resulting the combination of Coriolis induced stream mode and surface tension induced stream mode breakup. Observations of the breakup process for different volume flow rates and rotational speeds indicate that the breakup of liquid film stream is dependent on injection conditions and the corresponding cross flow velocity created by atomizer rotation. The breakup regime map is provided as a function of weber number and momentum flux ratio. Four distinct regimes are identified: Rayleigh breakup, bag breakup, multimode breakup, and shear breakup. The present results leads to understanding atomization performance and creating some idea to improved spray quality in this type of atomizer.
\end{abstract}

\section{Keywords}

Rotary atomizer, square shape injection orifice, high aspect ratio, discharge channel, Breakup regimes

\section{Introduction}

Rotary atomizer is one type of fuel injection which is used in small gas turbine engines. In big and popular gas turbine engines are used pressure atomized with a high-pressure fuel pump. The idea of using this type of fuel injectors system was raised to avoid the using of large and heavy pumps in small gas turbine engines. Higher rotational speed at which small gas turbine engines operation allow rotary atomizer to be used to create the fuel spray, replacing the high-pressure fuel pump. Actually, centrifugal force causes the fluid to be thrown from the discharge elements with high velocity. As the injection liquid film is introduced to environment, it gets converted into ligaments and sheets that eventually break into fine droplets. In this injectors, depending on design and application, the orifices may be arranged in a single row, multiple rows or other new arrangements. Simple design, light weight, low cost, decreasing engine size, high performance in spay atomization with high uniformity, and eliminating the high pressure pump are specifications of rotary atomization in small gas turbine engines. Other users also are conceivable for this injectors such as spray drying, spray coating, and etc. Which can be studied to operationalizing them.

There have been a little studies reported in the literature for rotary atomizers and since there is not existed the strong fundamental and theoretical base for this type of injectors yet to explains completely breaks up mechanism or design map. Design map is the important point to design and optimization for different scale of rotary atomizer in various operation conditions and this allows to sizing the combustor of small gas turbine engines or other conceivable application. There are parameters that must be examined such as size, shape, environment specification, fuel properties and holes injector geometry and dimension.

In this type of injectors, the fuel passes from the shaft and enters to the chamber, then discharges from the nozzles which are drilled on the fuel chamber. Dahm et al. [1] indicated that a thin liquid film is formed on the discharge channel wall and flows along it. Also Dahm proposed three injection modes including the regular film mode, irregular film mode, and the Coriolis-induced stream mode in liquid atomization. If this thin film flow can be maintained until it issues from the periphery of each channel, then the atomizer operates in the film-mode liquid breakup. In this type of injection mode, two phenomena can be observed: if the thin liquid film maintains its integrity to the channel exit and gets separated from the orifice edge, the atomizer demonstrates regular film mode injection. On the other hand, if the thin liquid film issues forth from the periphery of the hole pools on the atomizer surface rather than getting 
separated at the hole edge, the atomizer operates in the irregular film mode injection. The third injection mode, namely the Coriolis-induced stream mode, can occur when sufficiently large Coriolis forces act on the liquid flow within the channel and causes the liquid film to pull in to a side. Dahm in this study, attempted to introduces a basically theory for spray mechanism in the rotary atomization. Dahm et al. [2] provided flow visualization results with experimental setup to characterize the liquid breakup regimes involved in fuel rotary atomization over a range of hole geometries, hole sizes, and rotation rates. He was the only person in literature that is used the square hole shape to optimization of rotary atomization. Dahm observed the regular and irregular film mode injection in round radial discharge orifices. The visualization results indicate that in a regular film mode injection, the liquid film breaks up as three regimes subcritical, transition, and supercritical film mode breakups due to the competition between surface tension and inertia effects. In the subcritical regime, the liquid film is drawn together by surface tension to form a single liquid ligament that subsequently undergoes the classical Rayleigh breakup. If the thin liquid film breaks up into small ligaments and droplets soon after being introduced to the air, the atomizer operates in the supercritical film-mode breakup. They have also provided the proper length scale for correlating the atomization performance of the rotary atomizer with injection orifices operating in the subcritical and supercritical film-mode breakup regime [2]. For the rotary atomizer used in these researches, the length of the discharge channel is not long enough to make the Coriolis effect important. Therefore, Dahm no observation has been made for the Coriolisinduced stream mode injection for any shapes hole. In the following years, Choi et al. [3] elucidated the ignition and combustion characteristics of the slinger combustor with a rotating fuel injection system. They also provided the spray visualization of a rotary atomizer with round radial orifices and a clear correlation with drop size and ignition performance with rotational speed. They classified the breakup regimes to subcritical, transition, and supercritical modes without mentioning the Coriolis effect. Choi et al. [4] have measured the spray characteristics of a rotary atomizer with a radial-axial discharge channel by applying the Phase Doppler Particle Analyzer (PDPA) laser diagnostic technique. By using this system, the droplet size (SMD), velocity distribution, and spray pattern were measured for the first time. In this study, the breakup regimes were classified just like previous research studies. To determine the effect of the orifice injection diameter and the number of orifices, Choi et al. [5] constructed a test rig and measured droplet sizes as well as spray pattern visualization under various rotational speeds and liquid flow rates. They observed three spray patterns: ligament spray, regular spray, and irregular spray pattern; they found that there is an optimum orifice size for obtaining a minimum droplet size. No classification of breakup regimes was performed in the ligament spray pattern. This is equivalent to the Coriolis-induced stream mode breakup in this type of rotary atomizer. Most studies on the spray characteristics of the rotary atomizer have focused on droplet size measurement at relatively high rotational speeds. Sescu et al. [6] provided droplet size and volume distribution at a low rotational speed to analyze atomization performance in start-up phase conditions of small gas turbine engines with the rotary atomizer. The following year, Choi et al. studied the effect of rotational speed and air cross-flow velocity on droplet sizes [7] and spatial droplet distribution [8] around a rotary atomizer with round radial discharge orifices. They found that the cross-flow influences the Sauter mean diameter directly. However, the rotational speed has a large effect on the spray at slower cross-flow conditions. Paquet et al. [9] recently reviewed the rotary atomizer to find suitable correlations for an adequate spray distribution.

In this study is investigated breakup mechanism in a rotary atomizer with square shape discharge orifice and the breakup regime map is provided as a function of weber number and momentum flux ratio. The present investigation is used the one operational slinger rotary atomizer which the channel is long enough to observing the Coriolisinduced stream mode injection. Visualization experiments are conducted by high speed shadowgraph imaging technique with pulsed light illumination for the first time and also is observed the exact details from breakup regimes for the first time. Observations of the breakup process for different volume flow rates and rotational speeds indicate that the breakup of liquid film stream is dependent on injection conditions and the corresponding cross flow velocity created by atomizer rotation. Four distinct regimes are identified: Rayleigh breakup, bag breakup, multimode breakup, and shear breakup. The present results leads to understanding atomization performance and creating some idea to improved spray quality in this type of atomizer. The motivation of this study is the use of this type of orifice in some small gas turbine engines as well as non-existing observation in literature concerning about high aspect ratio of discharge channel. Dahm was the only person in literature that is used the square hole shape in rotary atomization which cannot shows Coriolis-induced stream mode injection and because of his experimental methods cannot shows the details of breakups regimes.

\section{Material and methods}

The experimental setup used for the current study was built at the SRMF optical laboratory of Sharif University of Technology. This setup has been designed to capture the spray behavior of a rotary atomizer in high rotational speeds. To provide realistic results, the current rotary atomizer is designed and built like the atomizers used in small gas turbine engines. All experimental tests were performed under ambient pressure and temperature $\left(20^{\circ} \mathrm{C}\right)$ using 
water as the test liquid in place of the aviation fuel to enhance safety. The liquid flow rate and the rotational speed are changed from $30 \mathrm{LPH}$ to $120 \mathrm{LPH}$ and $2,000 \mathrm{rpm}$ to $25,000 \mathrm{rpm}$, respectively. A schematic of the experimental setup and the rotary atomizer is shown in Figs. 1 and 2, respectively.

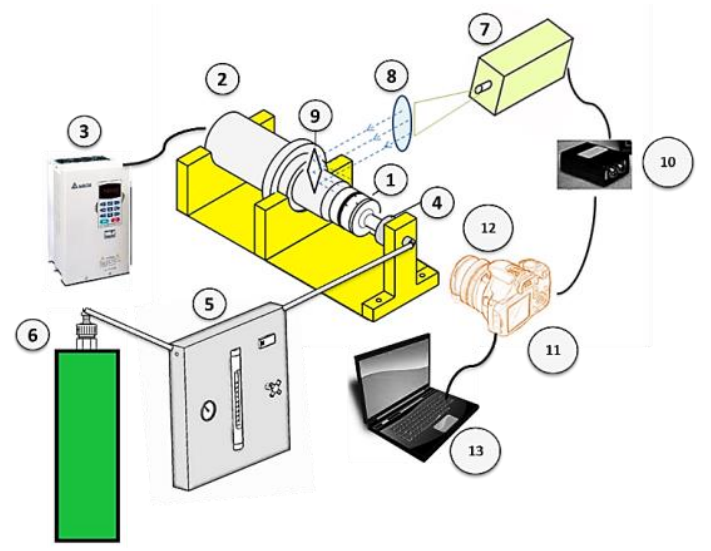

Figure 1: Components of the experimental setup: (1) rotary atomizer, (2) spindle motor, (3) variable-frequency drive, (4) bearing and housing, (5) flow control panel, (6) pressurized water tank, (7) pulse light, (8) Collimating lens, (9) mirror, (10) trigger, (11) camera, (12) macro lens, (13) laptop.

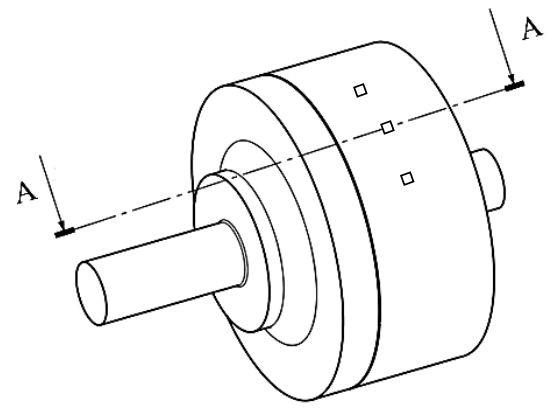

Isometric view

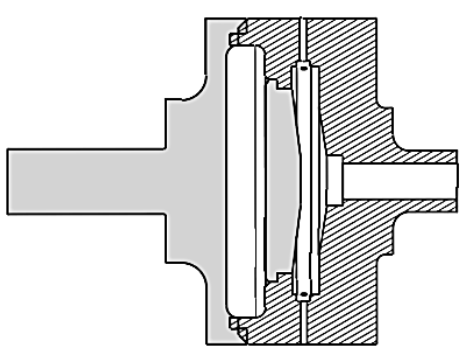

Section view A-A

Figure 2. Schematic of the rotary atomizer.

The experimental setup includes a high-speed spindle motor with $3.5 \mathrm{KW}$ power. The rotary atomizer is directly connected to this motor and rotates with a rotational speed up to $25,000 \mathrm{rpm}$. To adjust the frequency and rotational speed of spindle motor, a variable frequency drive of $5.5 \mathrm{KW}$ power is used. A high-speed angular contact-sealed bearing is mounted to the atomizer shaft. The liquid is fed to the atomizer disk through the hollow shaft designed with a labyrinth seal on the peripheral surface. As the liquid reaches the inner surface of atomizer, it spreads circumferentially and radially before being placed in the maximum radius of the atomizer cavity. The liquid film is discharged into the radial direction through the orifices and followed by its interaction with external air to form the ligaments or sheets that break into fine droplets. The rotary atomizer used in the current study consists of six square shapes injection orifices with width of $1.5 \mathrm{~mm}$. The atomizer was manufactured by CNC machining. The outer and inner diameters of the atomizer disk are $77.5 \mathrm{~mm}$ and $59 \mathrm{~mm}$, respectively and the cannels length are $9.25 \mathrm{~mm}$.

The flow control panel consists of the rotameter, pressure gage, and needle valve. The rotameter measures the flow rate of liquid within a range of $10 \mathrm{LPH}$ to $120 \mathrm{LPH}$ with an accuracy of $\pm 2 \mathrm{LPH}$. A needle valve was used in this panel to adjust the flow rate accurately. Before the needle valve is opened, the spindle motor runs and the atomizer speed reaches the steady speed. To avoid the variation of the volume flow rate during a test, water-pressurized tanks were used at a pressure of 3 bar to ensure that the upstream pressure of the needle valve is kept approximately constant. All the experimental results are taken after the flow rate of the liquid is kept fixed with fluctuations of less than $2 \mathrm{LPH}$. The pressure gauge with a range of $0.1-1$ bar was located upstream closer to the atomizer inlet to measure its pressure loss.

The optical setup consists of pulsed light illumination, collimating lens, mirror, trigger, camera, and macro lens. The pulse light is a high-power LED-based illuminator, which can produce green light pulses with a minimum duration of about $125 \mathrm{~ns}$. The pulse duration was set to $875 \mathrm{~ns}$ in the current work in both spray visualization and 
droplet size measurement. The collimating lens with a focal length of $40 \mathrm{~cm}$ was used to parallel the light beams emitted from the pulse light and to adjust the field of view. The mirror was located on the spindle front surface to change the direction of the light beams. As a result, the light beams align with the atomizer rotation axis and backilluminates the liquid jet emerging from atomizer orifices. Two HDSLR cameras, Nikon D3300 and D7100 are used to capture the breakup structure and droplets sampling, respectively. A trigger provides the possibility of synchronizing the emitted pulse with a camera shutter speed. The camera was operated with an open shutter in a dark room so that exposure times could be controlled by the duration of the light pulses. This pulse duration of 875 $\mathrm{ns}$ is sufficient to freeze the motion of the liquid jet structure in the high-speed rotation of the atomizer. The HDSLR cameras capture a high-resolution image with $6000 \times 4000$ pixels. The Nikon $200 \mathrm{~mm}$ f/4 ED macro lens with a working distance of about $26 \mathrm{~cm}$ was used to ensure the capture of small breakup structures and ligaments.

The shadowgraph technique with pulse back illumination is used for spray visualization. In this technique, a light source illuminates the liquid injection domain. The injected liquid obstructs the light beam and creates a shadow effect. By focusing the camera lens on the shadow plane, the resulting pattern is the picture of a fluid shadow whose contrast is recognizable from the background light. The camera focused at the plane of symmetry above the atomizer periphery surface in which the liquid jet emerges from injection orifices. The field of view is limited to $24 \mathrm{x}$ $36 \mathrm{~mm}$ and a reproduction ratio of 1:1 for spray visualization. This optical recording technique features low-cost low-power consumption and simple optical configurations compared to those of laser-based systems.

\section{Results and discussion}

As mentioned, in this present study is used the pulsed shadowgraph photography technique to Extracts the high resolution results in all range of operation in a rotary atomizer with square shape discharge orifice. The results of observations are unique and perfect. The effects of rotational speed and volume flow rate are studied on the breakup structure. As it is evident from the literature review, there is only one non-dimensional parameter (We) that describes the behavior of liquid jets and sprays by the rotary atomizer. The Weber number plays a significant role in multiphase flows, especially in case of drop formation. This non-dimensional parameter may be defined as the ratio of the inertial force to the surface tension force of the fluid. In the current study, the liquid to air momentum flux ratio, $q$, and the aerodynamic Weber number, Wed, are considered for the analysis. This is because the breakup mechanism primarily depends on the liquid injection velocity and the air cross-flow velocity. The liquid injection velocity can be varied by changing the mass flow rate in a constant rotational speed. Hence, it is not possible to carry out the analysis only by means of Wed. The liquid film thickness formed at the channel exit of slinger orifices is one of the key parameters relevant to its atomization properties. Dahm et al. [1] provided the following equation to calculate the liquid film thickness:

$\mathrm{t}=\left(\frac{3}{\pi}\right)^{1 / 3}\left(\frac{\mu_{\mathrm{L}} \mathrm{Q} / N}{\rho_{\mathrm{L}} R \Omega^{2} \mathrm{~d}}\right)^{1 / 3}$

Where $\mu_{\mathrm{L}}$ is the liquid viscosity, $\mathrm{Q}$ is the total volume flow, $N$ is the number of injection orifices, $\rho_{\mathrm{L}}$ is the liquid density, $R$ is the outer radius of atomizer disk, $\Omega$ is the rotational speed, and $\mathrm{d}$ is the injection orifice diameter. The liquid injection velocity and the air cross-flow velocity are obtained by Equations (2) and (3), respectively.

$\mathrm{V}_{\mathrm{a}}=\mathrm{R} \Omega$,

$\mathrm{V}_{\mathrm{l}}=\frac{\mathrm{Q}}{\mathrm{N} \pi \mathrm{dt}}$.

These velocities are then used for obtaining $\mathrm{q}\left(\rho_{\mathrm{L}} \mathrm{V}_{\mathrm{l}}^{2} / \rho_{\mathrm{a}} \mathrm{V}_{\mathrm{a}}^{2}\right)$. The aerodynamic Weber number based on the orifice diameter and the density and velocity of cross-flowing air, $V_{a}$, is defined as $W e_{d}=\rho_{a} V_{a}^{2} d / \sigma$. The liquid to air momentum flux ratio, q, and the aerodynamic Weber number, Wed, play a major role in primary and secondary atomization. The range of experimental boundary conditions and non-dimensional parameters used in the present work are listed in Table 1.

Table 1: Range of operating conditions and derived parameters used

\begin{tabular}{c|c} 
Rotational speed $(\Omega)$ & $2000-25000 \mathrm{rpm}$ \\
\hline Liquid volume flow rate $(\mathrm{Q})$ & $30-120 \mathrm{LPH}$ \\
Cross flow velocity $\left(V_{a}\right)$ & $12-101 \mathrm{~m} / \mathrm{s}$ \\
Liquid injection velocity $\left(V_{l}\right)$ & $4-45 \mathrm{~m} / \mathrm{s}$ \\
Aerodynamic Weber number $(\mathrm{We})$ & $2-170$ \\
Liquid to air momentum flux ratio $(\mathrm{q})$ & $25-674$ \\
Liquid film thickness (t) & $16-140 \mu \mathrm{m}$
\end{tabular}




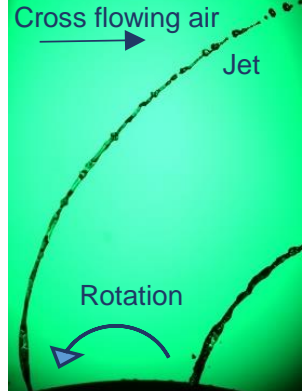

2000 rpm-30 LPH

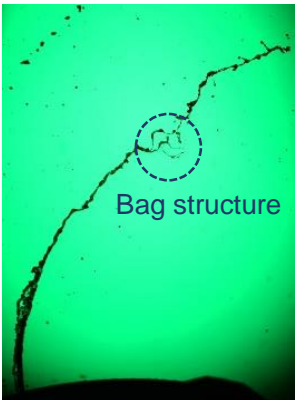

6000 rpm-30 LPH
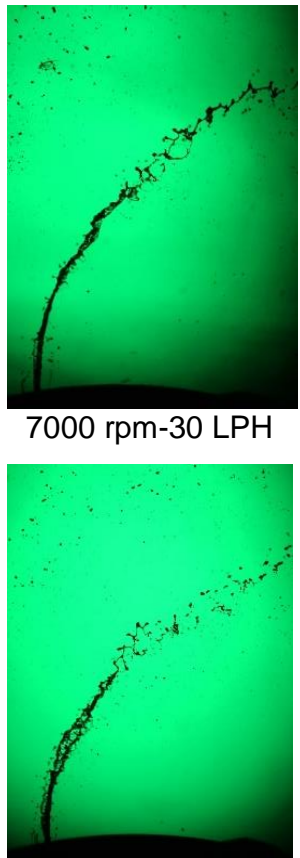

8000 rpm-30 LPH

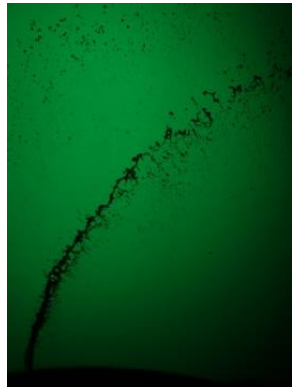

18000 rpm-30 LPH
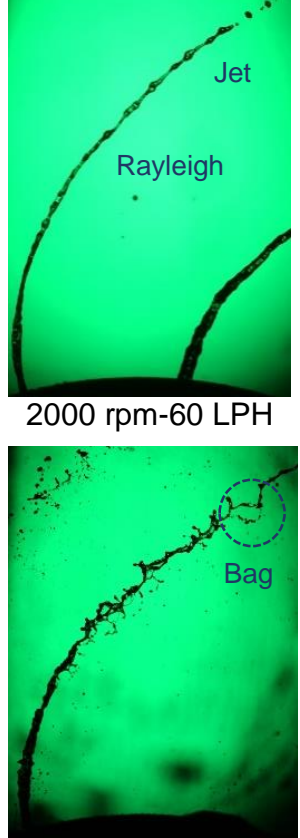

6000 rpm-60 LPH

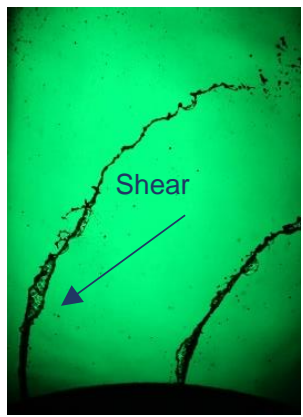

7000 rpm-30 LPH
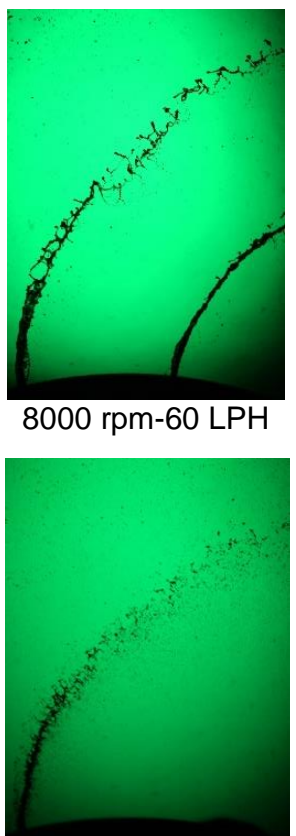

18000 rpm-60 LPH

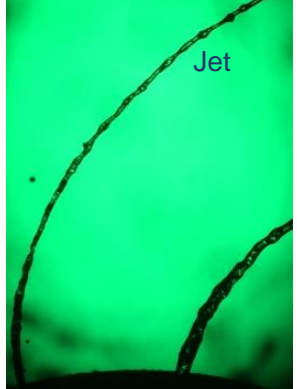

2000 rpm-90 LPH

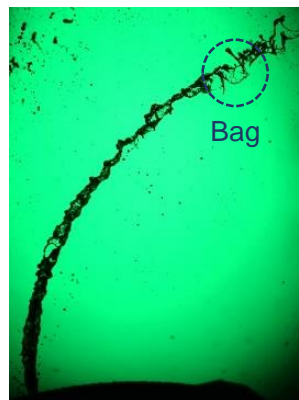

6000 rpm-90 LPH

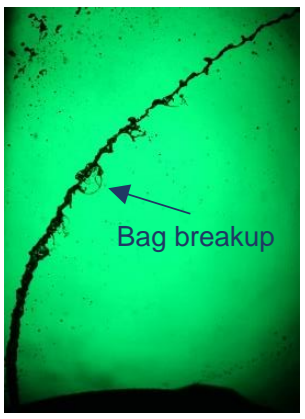

7000 rpm-30 LPH

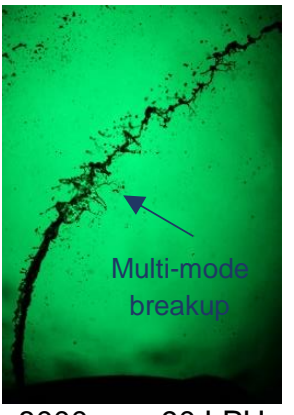

8000 rpm-90 LPH

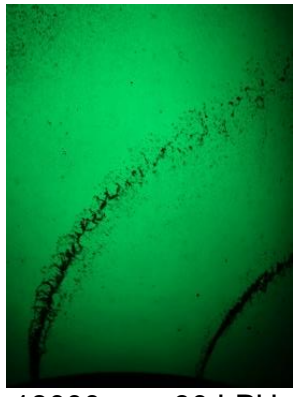

18000 rpm-90 LPH

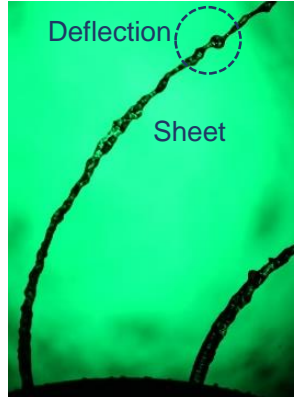

2000 rpm-120 LPH

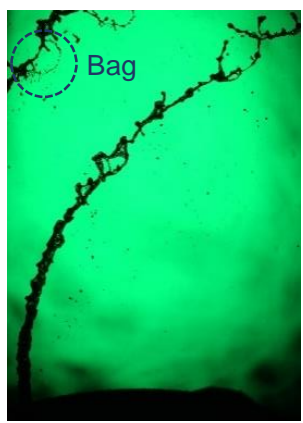

$6000 \mathrm{rpm}-120 \mathrm{LPH}$

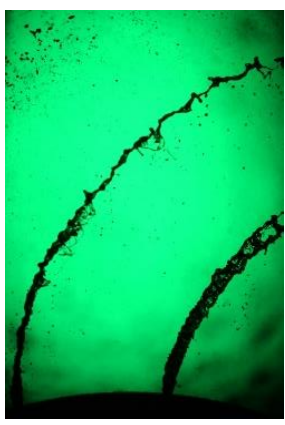

7000 rpm-30 LPH

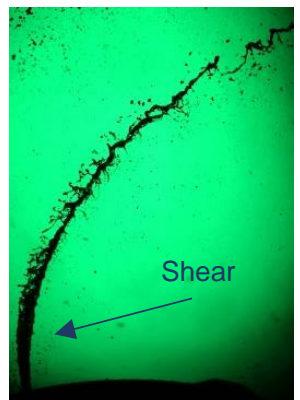

8000 rpm-120 LPH

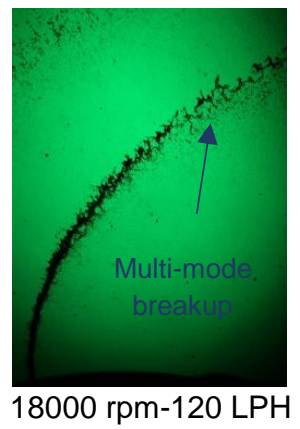

Figure 3. Spray visualization in rotational speed of $2,000,6,000,7,000,8,000$ and $18,000 \mathrm{rpm}$. 
For all cases considers in the present experiments, the liquid flow rate is kept constant at 30-120 LPH for six holes, so each holes liquid flow rate is $5-20 \mathrm{LPH}$ which is representative of practical fuel flow rates in small gas turbines and speeds ranging are 2000 to $25,000 \mathrm{rpm}$. Fig. 3 shows shadowgraph photos from side view of rotary atomizer and shows clear trends in breakup patterns as the rotational speed increases from 2000 to $18000 \mathrm{rpm}$ in 30 to 120 LPM. As mentioned, the rotational speed of rotary atomization equivalent of the cross flow velocity and the liquid fuel of rotary atomizer sprays in contras of cross flow velocity. Fig. 4 shows four distinct regimes are identified: Rayleigh breakup, bag breakup, multimode breakup, and shear breakup. First, the injected liquid stream at low rotational speed $(2,000 \mathrm{rpm})$ in all range of volume flow rates shows that the liquid jet is broken up in accordance with the Rayleigh mechanism. The Rayleigh jet breakup is identified by the growth of symmetric and asymmetric oscillations of the jet surface due to a lower inertial force. With increasing rotational speed and inertial force, the wave length of disturbance decreases. If the wave length of the initial disturbance is greater than the minimum wave length of disturbance, the surface tension force increases the disturbances and accordingly leads to disintegration of the liquid jet. Approximately the size of the droplets produced in this breakup regime are the same as that of the jet diameter.

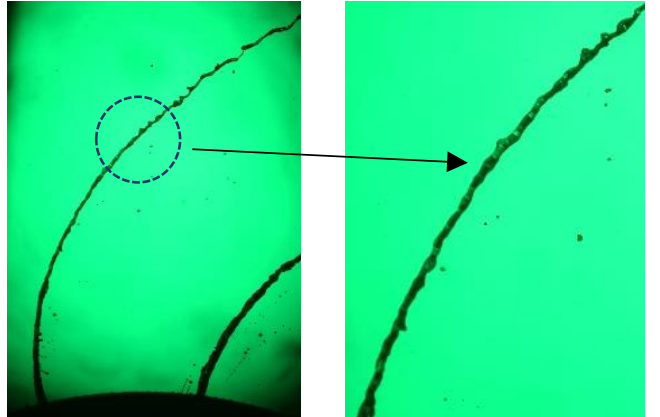

A) Rayleigh breakup regime (4000 rpm-90 LPH)

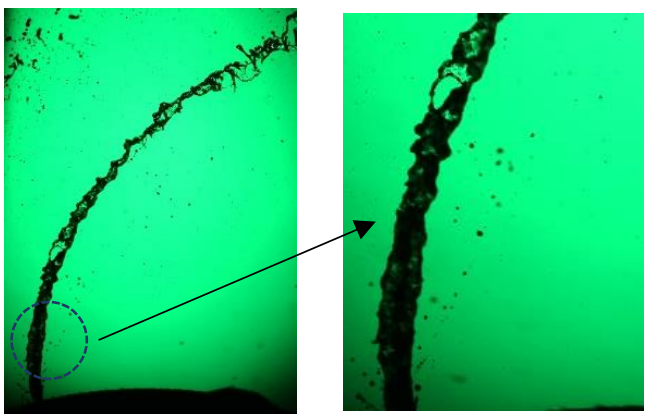

C) sheet breakup regime $(6000 \mathrm{rpm}-90 \mathrm{LPH})$

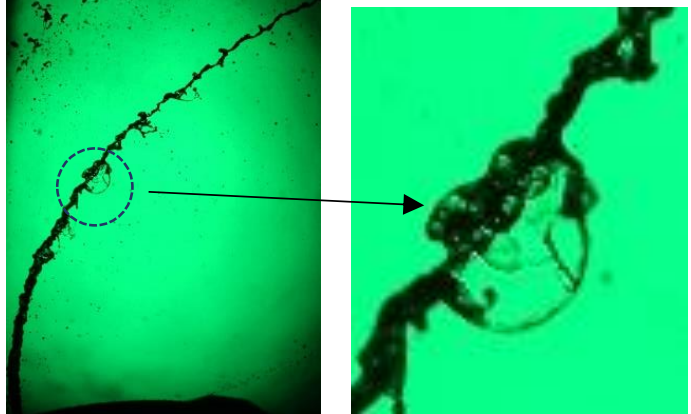

B) Bag breakup regime (7000 rpm-30 LPH)

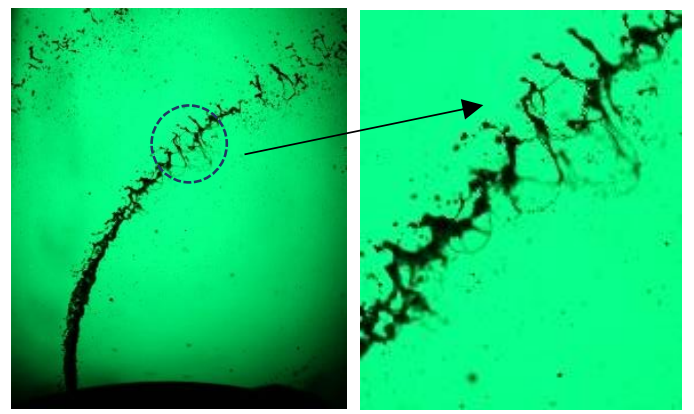

D) multimode breakup regime (6000 rpm-30 LPH)

Figure 4. Four distinct regimes are identified: Rayleigh breakup, bag breakup, and shear breakup, multimode breakup.

Second, next breakup mechanism, which was observed at a rotational speed of 6,000 rpm with a volume flow rate of $30 \mathrm{LPH}$, is bag breakup (fig.3). The bag structures were also observed in other operating conditions such as 7,000 and $8,000 \mathrm{rpm}$ with volume flow rates of $90 \mathrm{LPH}$, among others. The bag structures are formed by increasing the distance between the nodes to allow the gas to be permeated into the spacing between them and mixed with the liquid. The reason of forming these structures on the liquid stream surface is that the liquid film issued from the atomizer orifice deflects in the stream-wise direction, and the timescale of the crossflow velocity is lower than that of the liquid injection. Hence, the crossflowing air has enough time to permeate into the liquid between nodes and to create bag structures.

Third, the other phenomenon, which can be observed in a volume flow rate of $120 \mathrm{LPH}$ at different rotational speeds, is the thin liquid sheet that is formed after the liquid film is injected into the environment (fig.3). This behavior can be explained by comparing two timescales. If the timescale of surface tension is lower than that of the liquid injection inertial force, then the liquid film collapses to a single liquid column as a form of liquid jet. Otherwise, the surface tension has not enough time to exert any effect on the liquid film and hence the film is spread into the spanwise direction as a form of the thin liquid sheet.

Fourth, the last breakup mechanism is multimode regime which can be observed in volume flow rate of $30 \mathrm{LPH}$ at $6000 \mathrm{rpm}$, volume flow rate of $60 \mathrm{LPH}$ at $8000 \mathrm{rpm}$ and etc. (fig.3). 
Dahm et al. [2] introduced the classification for all range of rotary atomizer operation. The visualization results indicate that the liquid film breaks up as three regimes subcritical, transition, and supercritical film mode breakups due to the competition between surface tension and inertia effects. In the subcritical regime, the liquid film is drawn together by surface tension to form a single liquid ligament that subsequently undergoes the classical Rayleigh breakup. If the thin liquid film breaks up into small ligaments and droplets soon after being introduced to the air, the atomizer operates in the supercritical film-mode breakup. They have also provided the proper length scale for correlating the atomization performance of the rotary atomizer with injection orifices operating in the subcritical and supercritical film-mode breakup regime [2]. In this study by definition is provided Dahm, the breakup regime map is provided as a function of weber number and momentum flux ratio in for the first time (fig. 5).

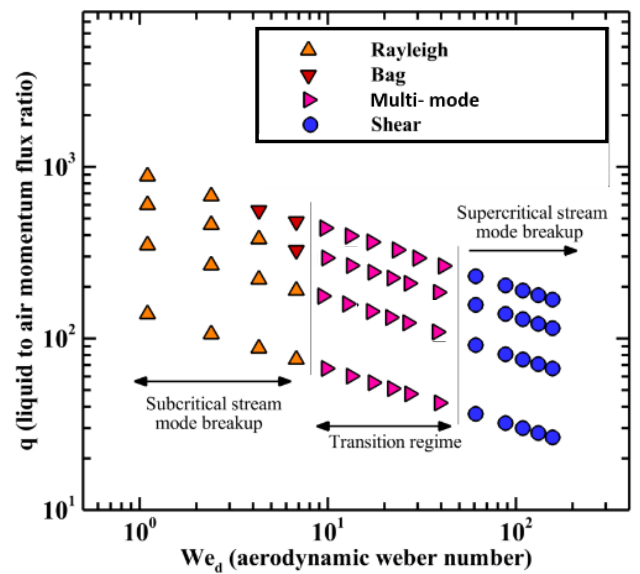

Figure 5. The breakup regime map for the rotary atomizer with the square discharge orifice

Dahm et al. [1] indicated that a thin liquid film is formed on the discharge channel wall and flows along it. Also Dahm proposed three injection modes including the regular film mode, irregular film mode, and the Coriolis-induced stream mode in liquid atomization. In Dahm study, the length of the discharge channel is not long enough to make the Coriolis effect important. Therefore, Dahm no observation has been made for the Coriolis-induced stream mode injection for any shapes hole. In this study, the effects of a high aspect ratio noncircular discharge channels are considered. The motivation of this study is the use of this type of orifice in some small gas turbine engines as well as non-existing observation in literature concerning about high aspect ratio of discharge channel. The visualizations indicates that the liquid film formed along the channel is pushed to one side of it due to Coriolis force which is dominant in this type of atomizer. The liquid film is accumulated on one side of the discharge channel and emerges from the atomizer orifices as a crescent-shaped form. Fig. 6 shows an enlarged view of the liquid film formation at the end of the channel. The Coriolis force acts on the liquid film and it is pushed to one side of the channel opposing the rotation. This observation shows the Coriolis force is stronger than surface tension force which was created by corners of square orifice.

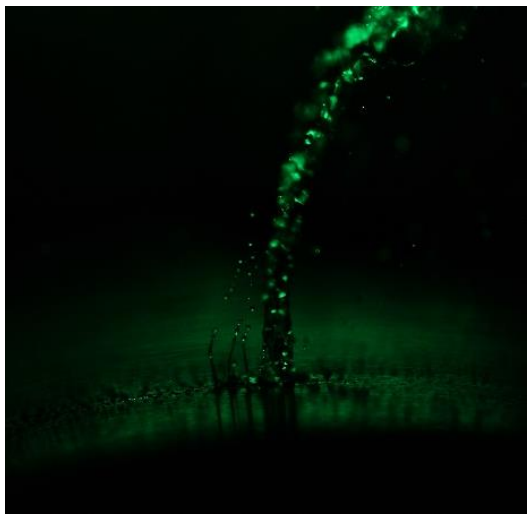

A) liquid injection in square hole's root (1000 rpm-10 LPH)

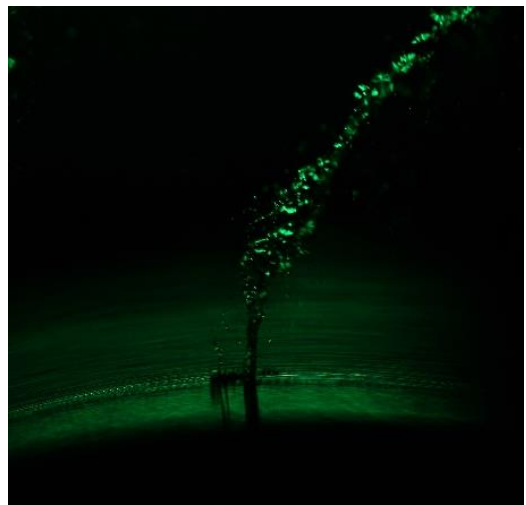

B) liquid injection in square hole's root (2000 rpm-30 LPH)

Figure 6. Liquid injection in square hole's root 


\section{Conclusions}

An experimental investigation of breakup mechanism in a rotary atomizer with square shape discharge orifice operating in the Coriolis-induced stream mode injection over a range of rotational speeds and volume flow rates. The dynamics of the interaction between the air cross-flow and the thin liquid film is explained through flow visualization experiments. Four different regimes of spray breakup are identified: Rayleigh breakup, bag breakup, multimode breakup, and shear breakup. A breakup regime map is presented at We on abscissa and q on ordinate. The results suggest qualitative similarities between the primary breakups of the thin liquid film in this type of atomizer, the primary breakup of the non-turbulent liquid jet, and the fanlike liquid sheet in a cross flow. This observation shows the Coriolis force is stronger than surface tension force and the liquid film formed along the channel is pushed to one side of it due to Coriolis force which is dominant in this type of atomizer. The present results leads to understanding atomization performance and creating some idea to improved spray quality in this type of atomizer.

\section{References}

[1] Dahm, W. J. A., Patel, P. R \& Lerg, B. H., Analysis of liquid breakup regimes in fuel slinger atomization, Atomization and Sprays, vol. 16, no. 8, pp. 945-962, 2006.

[2] Dahm, W. J. A., Patel, P. R \& Lerg, B. H., Experimental visualizations of liquid breakup regimes in fuel slinger atomization, Atomization and Sprays, vol. 16, no. 8, pp. 933-944, 2006.

[3] Choi, S., Lee, D. \& Park, J., Ignition and combustion characteristics of the gas turbine slinger combustor, J. Mech. Sci. Technol., vol. 22, no. 3, pp. 538-544, Mar. 2008.

[4] Choi, S. M., Jang, S. H., Lee, D. H. \& You, G. W., Spray characteristics of the rotating fuel injection system of a micro-jet engine, J. Mech. Sci. Technol., vol. 24, no. 2, pp. 551-558, Feb. 2010.

[5] Jang, S. H. \& Choi, S. M., Spray behavior of the rotary atomizer with In-line Injection Orifices, Atomization and Sprays, vol. 20, no. 10, pp. 863-875, 2010.

[6] Sescu, C., Kucinschi, B. R., Afjeh, A. A. \& Masiulaniec, K. C., Experimental test rig with results on liquid atomization by Slinger injectors, Journal of Engineering for Gas Turbines and Power, vol. 133, no. 11, p. 114505, 2011.

[7] Choi, S. M., Yun, S., Jeong, H. J. \& Corber, A., Spray in cross flow of a rotary atomizer, Atomization and Sprays, vol. 22, no. 2, pp. 143-161, 2012.

[8] Choi, S. M., Yun, S., Jeong, H. J. \& Corber, A., Spatial drop behavior of a rotary atomizer in a cross flow, Atomization and Sprays, vol. 22, no. 12, pp. 1077-1095, 2012.

[9] Paquet, B., de Champlain, A. \& Kalla, S., Review of fuel spray distributions to predict performance of rotary atomizers in a slinger gas turbine combustor, Atomization and Sprays, vol. 26, no. 5, pp. 483-511, 2016. 